\title{
Níveis de autocontrole em alunos de cursos à distância e presencial e sua relação com os objetivos acadêmicos
}

A falta de motivação diante da responsabilidade, quanto ao controle do seu aprendizado, é um dos motivos para a evasão de alunos. Em ambientes de educação à distância (EAD), existe a expectativa de que os alunos possam realizar grande parte do processo de aprendizagem. Assim, o autocontrole teria papel central no sucesso acadêmico. Utilizar estratégias de autocontrole pode levar a maiores índices de sucesso, especialmente em pessoas com tendências à procrastinação. $O$ presente trabalho analisou o perfil de autocontrole de alunos de ensino presencial e à distância, para verificar se estes apresentam diferentes perfis e se maiores índices de autocontrole levam os alunos a alcançarem seus objetivos acadêmicos. Foi possível identificar, a partir do teste de comparação de médias MannWhitney-U, que os grupos de alunos do ensino presencial e à distância apresentam diferentes médias em relação ao autocontrole, tendo os alunos do ensino à distância maiores índices e, através do teste de correlação de Spearman, identificou-se que maiores índices de autocontrole levam ao alcance dos objetivos acadêmicos.

Palavras-chave: Autocontrole; Objetivos acadêmicos; Ensino à distância.

\section{Levels of self-control in distance and classroom students and their relationship with academic goals}

\begin{abstract}
Lack of motivation in the face of responsibility for controlling their learning is one of the reasons for student dropout. In distance education (ODL) environments, students are expected to be able to do much of the learning process. Thus, self-control would play a central role in academic success. Using self-control strategies can lead to higher success rates, especially in people with procrastination tendencies. The present work analyzed the self-control profile of classroom and distance learning students, to verify if they present different profiles and if higher self-control indices lead the students to reach their academic goals. It was possible to identify, from the Mann-Whitney-U comparison test, that the groups of students in classroom and distance education present different means in relation to selfcontrol, having students of distance education higher indexes and, through the Spearman's correlation test found that higher self-control indices lead to the achievement of academic goals.
\end{abstract}

Keywords: Self-control; Academic goals; Distance learning.

Topic: Inovação Tecnológica

Reviewed anonymously in the process of blind peer
Received: 10/07/2019

Approved: 12/09/2019
Humberto Caetano Cardoso da Silva (ID Universidade Federal de Pernambuco, Brasil http://lattes.cnpq.br/4594928852071554 http://orcid.org/0000-0001-9584-4465 humberto.ccs@gmail.com

Filype Rosas de Morais

Centro Universitário Maurício de Nassau, Brasil http://lattes.cnpq.br/1846510246899296

filypemorais@gmail.com
Referencing this:

SILVA, H. C. C.; MORAIS, F. R.. Níveis de autocontrole em alunos de cursos à distância e presencial e sua relação com os objetivos acadêmicos. Revista Brasileira de Administração Científica, v.10, n.3, p.97-105, 2019. DOI: http://doi.org/10.6008/CBPC2179$684 \times .2019 .003 .0008$ 


\section{INTRODUÇÃO}

De acordo com o Relatório analítico de aprendizagem à distância no Brasil, em 2017, havia mais de 1,7 milhão de alunos matriculados em cursos à distância ou semipresenciais. Entretanto, os níveis de evasão são, também, consideráveis. Para Neves (2018), o índice de evasão em instituições particulares de ensino superior chegou a 32,5\% em 2014. Adicionalmente, para Silva et al. (2011), a evasão dos alunos está relacionada a fatores como a falta de motivação diante da responsabilidade quanto ao controle da sua autoaprendizagem e a rarefeita relação com os professores.

Na literatura sobre educação à distância, a expectativa de que os alunos possam realizar grande parte do processo de aprendizagem por conta própria, de forma independente, é um tema bastante presente (BAYNTON, 1992; ZHU et al., 2016; DUCKWORTH, 2019). Entretanto, Baynton (1992) argumenta que o conceito de independência não representa, adequadamente, a complexidade de interações e variáveis presentes no processo de educação a distância, propondo, assim, que o conceito de controle seria melhor empregado. Para Wolfe et al. (1995), o auto controle é o preditor mais importante, entre as 32 variáveis de personalidade do Jackson Personality Inventory (JPI) (JACKSON, 1997), do alcance dos objetivos acadêmicos dos estudantes.

No contexto de psicologia da personalidade, o termo 'autocontrole' é utilizado para se referir a habilidade de realizar planos e cumpri-los face a dificuldades e desafios (BOL et al., 2011; ZHU et al., 2016). Nesta linha, Bol et al. (2011) argumenta que alunos de ensino à distância enfrentam problemas ainda maiores que alunos de ensino presencial. Para Bol et al. (2011), os alunos enfrentam a natureza extremamente autônoma do ensino à distância e a falta do suporte presencial que um professor provê no ensino presencial.

Assim, o autocontrole pode contribuir com o sucesso dos alunos no processo de aprendizagem (KOMARRAJU et al., 2013; ZHU et al., 2016). Entretanto, segundo Ariely et al. (2002) o autocontrole também é importante para alunos de ensino presencial. Assim, aqueles que se impõem prazos e objetivos tendem a ter maior sucesso acadêmico.

O alcance dos objetivos acadêmicos dos estudantes de cursos de graduação é uma preocupação das organizações de ensino superior (SCHUNK et al., 2012). Vários são os autores que afirmam que o autocontrole desempenha papel central no alcance do objetivos acadêmicos de aluno que cursam graduações na modalidade ensino à distância (BOL et al., 2011; SCHUNK et al., 2012). Adicionalmente, utilizar estratégias de autocontrole, como a utilização de prazos auto impostos, pode levar a maiores índices de sucesso, especialmente em pessoas com tendências a procrastinação (ARIELY et al., 2002). Assim, o presente trabalho tem como objetivo verificar os diferentes níveis de autocontrole existentes em grupos de estudantes de ensino à distância e ensino presencial e a relação entre o autocontrole e o alcance dos objetivos acadêmicos de alunos de graduação.

A proposição feita no presente estudo é de que alunos de ensino à distância apresentam perfil com maiores índices de autocontrole que alunos matriculados em cursos de graduação na modalidade presencial. Assim, estudar como se relacionam essas variáveis poderá trazer nova luz ao fenômeno, possibilitando 
melhores índices de retenção de alunos no contexto do ensino superior à distância.

Diante do contexto apresentado, a presente pesquisa será norteada pela pergunta central: Quais os níveis de autocontrole de alunos de graduação na modalidade de ensino à distância e da modalidade presencial? Visando à condução da presente pesquisa, foram analisados dados provenientes de alunos de cursos de educação à distância e alunos de educação presencial matriculados em instituições de ensino superior situadas no nordeste brasileiro.

\section{REVISÃO TEÓRICA}

Nesta seção será iniciada a discussão teórica sobre os objetivos acadêmicos, o autocontrole e o papel do professor-tutor na condução do processo de aprendizagem no contexto de cursos de graduação na modalidade ensino à distância. A seção será encerrada com o modelo a ser estudado e as hipóteses a serem testadas na pesquisa.

\section{Objetivos acadêmicos}

O alcance dos objetivos acadêmicos está relacionado com uma série de consequentes positivos como maior probabilidade de conseguir uma vaga no mercado de trabalho, maiores chances de admissão em programas de educação stricto sensu e maiores salários (DIXSON, 2018; SINGH et al., 2019). Para Singh et al. (2019), as principais métricas utilizadas para avaliar o alcance dos objetivos acadêmicos pode ser o Grade Point Average (GPA), bastante utilizado em países de língua inglesa. No Brasil, são utilizadas medidas como o Exame Nacional do Ensino Médio (ENEM).

Entretanto, essas medidas são aplicáveis aos alunos que se encontram no ensino médio. Para alunos de graduação, tanto Dixson (2018), quanto Singh et al. (2019) afirmam que a média das notas obtidas no decorrer do curso universitário, possibilita uma boa imagem da evolução acadêmica do estudante. Para o presente trabalho, foi utilizada a média das notas obtidas no semestre da coleta de dados. Para fins de comparação, estas foram normalizadas.

\section{O autocontrole}

Para Bayton (1992) o conceito central na condução de cursos de educação à distância é o controle. Sendo o autocontrole o preditor mais importante para o alcance dos objetivos acadêmicos (WOLFE et al., 1995), este será o construto avaliado no presente estudo. Para Kuhlmann (2012) as formas de controle podem ser classificadas em formais e informais. Os mecanismos de controle formais são divididos em estruturais, como a existência de professores, monitores e pessoal de apoio nas escolas, e processuais, como o próprio processo de aula, chamadas e avaliações. Já os mecanismos informais dividem-se em controle do grupo, no qual os próprios membros de um grupo estabelecem qual o comportamento aceitável, e autocontrole.

Autocontrole pode ser definido como uma regulação autoiniciada de pensamentos, sentimentos e ações quando o aluno enfrenta situações conflitantes entre objetivos de longo prazo e gratificações 
momentárias (DUCKWORTH, 2019). Conforme demonstrado na figura 1, em momentos de conflito, o aluno tem a escolha de realizar a tarefa ou buscar prazer imediato. Entretanto, o autocontrole possibilitaria que o aluno, busque realizações de mais longo prazo.

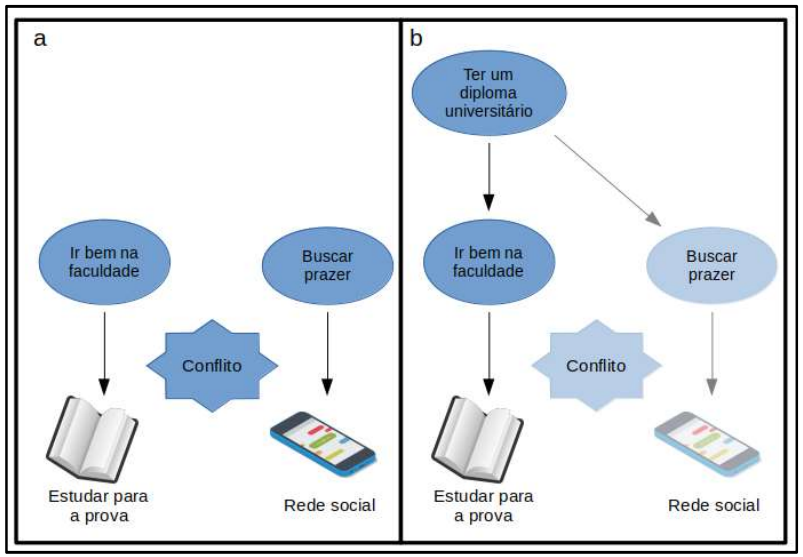

Figura 1: Autocontrole e conflitos. Fonte: Duckworth et al. (2019).

Para Tangney et al. (2004), a habilidade de inibir impulsos de forma que objetivos de maior valor a longo prazo tem sido a marca do mundo civilizado. Nesta linha, Komarraju et al. (2013) afirma que o autocontrole tem a capacidade de contribuir com o sucesso dos alunos no processo de aprendizagem, pois estes estariam mais propensos a vislumbrar recompensas de longo prazo e não gratificação imediata.

\section{Hipóteses de Pesquisa e Modelo Teórico Proposto}

A partir do exposto, apresentar-se-ão as hipóteses que nortearão o presente estudo: Hipótese 1: Os alunos matriculados em cursos de graduação na modalidade à distância possuem maiores índices de autocontrole que alunos matriculados na modalidade presencial; e Hipótese 2: O autocontrole tem relação positiva com o alcance dos objetivos acadêmicos de alunos matriculados em cursos de graduação. Adicionalmente, a figura 2 apresenta o modelo teórico a ser utilizado na validação das hipóteses do presente estudo.

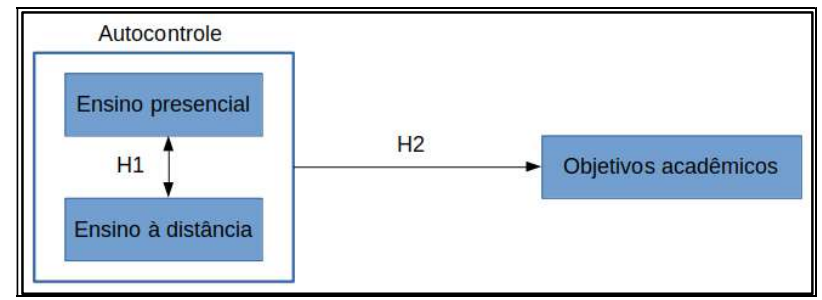

Figura 2: Modelo teórico proposto.

\section{METODOLOGIA}

O presente capítulo traz uma visão geral dos aspectos metodológicos que orientam esta investigação. Primeiramente vai ser apresentada a caracterização da pesquisa, os procedimentos de coleta e a análise dos dados. Esta pesquisa utilizou os pressupostos sugeridos por Hair (2009) e Malhotra et al. (2017), para estudos de natureza quantitativa, caracterizando esta pesquisa como descritiva, com a utilização de um levantamento (survey), com corte transversal único. 
A pesquisa foi realizada com alunos de graduação de variados cursos de instituições de ensino superior situadas no nordeste brasileiro. A amostra utilizada no estudo foi obtida por conveniência. Segundo Hair (2009), o tamanho mínimo da amostra depende do número de variáveis preditoras que estão sendo consideradas na análise. Para que seja realizada a etapa de análise fatorial para validação da escala proposta por Ferguson et al. (2006), Hair (2009) indica que, no mínimo, existam 5 (cinco) casos para cada variável. Entretanto, em amostras com poucos casos existe a possibilidade de relações entre as variáveis não serem detectadas, sendo indicado, pelo menos, 100 casos (HAIR, 2009). Assim, para o presente estudo, será assumida a amostra mínima de 100 casos.

\section{Variáveis Pesquisadas}

De acordo com o modelo teórico apresentado na figura 2, três construtos foram abordados e testados. O construto 'objetivos acadêmicos' foi analisado a partir das notas obtidas pelos alunos ao final do semestre. Na tabela 1, estão apresentadas as variáveis que foram pesquisadas através da survey. Os trabalhos que serviram como referência para a criação do instrumento de pesquisa também estão apresentados na mesma tabela. $O$ instrumento de coleta de dados foi finalizado com a utilização de questões para o levantamento de informações a fim de caracterizar o perfil dos entrevistados.

Tabela 1: Resumo com escalas utilizadas por construto.

\begin{tabular}{|l|l|l|l|}
\hline \multicolumn{1}{|c|}{ Construtos } & \multicolumn{1}{c|}{ Itens de Escala } & Questões & \multicolumn{1}{c|}{ Escalas Utilizadas } \\
\hline Autocontrole & AUTC_1 a 13 & 1 a 13 & (TANGNEY et al., 2004) \\
\hline
\end{tabular}

\section{Validação do Instrumento de Coleta de Dados}

Após elaborado o instrumento de coleta de dados, o mesmo foi submetido a uma avaliação conhecida como validade de face (KINNEAR et al., 1987) ou validade nominal (MALHOTRA et al., 2017). Posteriormente, foram realizados testes de dimensionalidade e confiabilidade (HAIR, 2009). $O$ instrumento de pesquisa foi submetido, então, a um pré-teste, de forma que possíveis problemas pudessem ser identificados. Assim, 10 alunos realizaram o preenchimento do questionário na fase pré-teste, sendo o tempo médio de preenchimento de 3 minutos e sem que nenhum problema fosse apontado.

Participaram da pesquisa alunos dos cursos presenciais de Administração, Ciências Contábeis, Enfermagem, Licenciatura em Letras, Pedagogia e Psicologia. Já os alunos de cursos à distância estavam distribuídos nos cursos de Administração Pública, Análise e Desenvolvimento de Sistemas, Licenciatura em Computação, Licenciatura em Física, Licenciatura em História, Licenciatura em Letras, Pedagogia e Sistemas de Informação.

\section{Procedimento de análise de dados}

Para a análise dos dados da presente pesquisa, além da avaliação dos dados de estatística univariada, foram utilizadas as técnicas multivariadas de análise fatorial exploratória (HAIR, 2009), de comparação de grupos Mann-Whitney-U (RUXTON, 2006) e de correlação de Spearman (HAUKE et al., 2011). Para o presente estudo, levando-se em consideração o tipo de dado ordinal coletado e sua característica não paramétrica, 
optou-se pela utilização do teste não paramétrico de comparação de grupos Mann-Whitney-U (RUXTON, 2006).

Já a análise das relações das variáveis foi realizada com o coeficiente de correlação de Spearman, que é um teste não paramétrico que mede a força da associação entre duas variáveis. Diferentemente do coeficiente de correlação de Pearson, não são requeridos associação linear entre as variáveis, nem que as variáveis envolvidas na avaliação sejam do tipo escalares (HAUKE et al., 2011). Assim, a utilização do referido coeficiente de correlação se justifica, apesar de contar, no presente estudo, com uma variável escalar, as medidas realizadas para avaliar o perfil de autocontrole dos estudantes são ordinais.

\section{RESULTADOS E DISCUSSÃO}

Na presente seção, serão analisados os dados obtidos a partir da survey. Apresentar-se-ão as estatísticas descritivas, caracterizando, assim, a amostra. Posteriormente, serão apresentadas as análises multivariadas e testes estatísticos, possibilitando a confirmação ou não das hipóteses apresentadas no estudo.

A pesquisa foi realizada no mês de maio de 2019 , junto à alunos de graduação de cursos presenciais e à distância. Participaram da pesquisa alunos dos cursos presenciais de Administração, Ciências Contábeis, Enfermagem, Licenciatura em Letras, Pedagogia e Psicologia. Já os alunos de cursos à distância estavam distribuídos nos cursos de Administração Pública, Análise e Desenvolvimento de Sistemas, Licenciatura em Computação, Licenciatura em Física, Licenciatura em História, Licenciatura em Letras, Pedagogia e Sistemas de Informação.

A caracterização da amostra será apresentada em termos de frequências relativas. Entre os respondentes, $56,9 \%$ são do sexo feminino e $43,1 \%$ do sexo masculino. A faixa etária dos participantes da pesquisa varia de 17 a 59 anos, sendo que $50 \%$ dos participantes encontram-se na faixa até 24 anos. Em relação aos cursos de graduação frequentados, o que apresentou maior frequência foi o de Enfermagem, com $16,9 \%$ do total de respondentes. Em seguida, aparecem os cursos de Psicologia, com 15,6\%, e Análise e Desenvolvimento de Sistemas, com 14,4\%. Finalmente, entre os participantes da pesquisa, 56,9\% são alunos de cursos presenciais, enquanto $43,1 \%$ são alunos de cursos à distância. Os dados referentes ao tipo de curso estão na tabela 2.

Tabela 2: Tipo de curso da amostra pesquisada.

\begin{tabular}{|l|c|c|}
\hline Tipo de Curso & Quantidade & Frequência Relativa \\
\hline Presencial & 91 & $5,9 \%$ \\
\hline À Distância & 69 & \\
\hline Total & 160 & \\
\hline
\end{tabular}

\section{Confiabilidade do instrumento}

A análise da confiabilidade do instrumento foi realizada a partir do cálculo do coeficiente alfa de Cronbach. Para o presente estudo e de acordo com as recomendações para estudos quantitativos, valores superiores a 0,7 indicam boa consistência interna da escala (HAIR, 2009). Para o presente estudo o valor calculado do coeficiente alfa de Cronbach foi de 0,807 . A tabela 10 possui os valores do coeficiente caso os 
itens sejam removidos, mostrando que existe uma boa consistência interna na escala.

A escala utilizada no presente estudo foi obtida a partir da escala de autocontrole proposta por Tangney et al. (2004). Visando, então, verificar a dimensionalidade das questões foi conduzida uma análise fatorial exploratória (HAIR, 2009). A variância explicada para o modelo foi de 58,59\%, o teste KMO obteve valor de 0,831 e o teste de esfericidade de Bartlett obteve $p<0,001$, indicando boa adequação dos dados para a análise fatorial. As quatro dimensões propostas por Costa et al. (1992), impulsividade, autodisciplina, foco e escolhas, apresentaram-se nos resultados. A análise fatorial está disponível na tabela 3.

Tabela 3: Confiabilidades calculadas da escala de autocontrole.

\begin{tabular}{|c|c|c|c|c|}
\hline Item da escala & 1 & 2 & 3 & 4 \\
\hline Eu sou bom em resistir à tentação & ,755 & 025 & 047 & ,206 \\
\hline Eu tenho dificuldade em quebrar maus hábitos* & ,730 & 094 & ,316 &,- 060 \\
\hline Eu sou preguiçoso* & ,710 & 156 & 051 & ,273 \\
\hline Eu digo coisas inapropriadas* & 628 & 191 & 078 &,- 315 \\
\hline Eu faço certas coisas que são ruins para mim, se são divertidas* & ,574 &,- 072 & ,286 & 078 \\
\hline Eu recuso as coisas que são ruins para mim & ,515 & 312 & ,347 &,- 161 \\
\hline Eu gostaria de ter mais autodisciplina* & 067 & ,723 & 000 & 200 \\
\hline As pessoas diriam que eu tenho autodisciplina de ferro &,- 061 & ,707 & ,201 & 087 \\
\hline Prazer e diversão às vezes me impedem de trabalhar* & ,356 & 678 & ,042 &,- 091 \\
\hline Eu tenho dificuldade em me concentrar* & 193 &,- 001 &, 818 & ,307 \\
\hline Eu sou capaz de trabalhar efetivamente para objetivos de longo prazo & 112 & 161 & 602 &,- 427 \\
\hline Às vezes não consigo me impedir de fazer algo, mesmo sabendo que está errado* & ,372 & ,215 & ,533 & -117 \\
\hline Costumo agir sem pensar em todas as alternativas* & , 165 & ,304 & 030 & ,761 \\
\hline
\end{tabular}

\section{Teste Mann-Whitney U}

O teste Mann-Whitney $U$ foi utilizado para avaliar a diferença entre os grupos de estudantes de ensino superior nas modalidades presencial e à distância. A hipótese 1 do presente estudo estipula que estes grupos são diferentes em relação às dimensões do autocontrole. A partir do teste, é possível identificar que os grupos, alunos de ensino presencial e alunos de ensino na modalidade à distância, apresentam, estatisticamente, diferença nas dimensões impulsividade, autodisciplina e foco. Os resultados dão suporte a hipótese 1 do estudo.

Dessa forma, o presente estudo corrobora com a indicação que alunos de ensino à distância tem no autocontrole estratégia central no alcance dos objetivos acadêmicos (BOL et al., 2011; SCHUNK et al., 2012). Os valores da estatística podem ser observados na tabela 4. Ao serem avaliadas as médias dos dois grupos do estudo, é possível observar que os alunos do ensino à distância possuem maiores valores de autocontrole em todas as dimensões do estudo. A tabela 5 exibe a comparação das médias entre os dois grupos.

Tabela 4: Comparação de grupos pelo teste Mann-Whitney U.

\begin{tabular}{|c|c|c|c|c|}
\hline & Impulsividade & Autodisciplina & Foco & Escolhas \\
\hline Mann-Whitney U & 2008,5 & 2341,5 & 2555,5 & 2739,5 \\
\hline$Z$ & $-3,938$ & $-2,804$ & $-2,037$ & $-1,434$ \\
\hline Sig. & $0,000^{*}$ & $0,005^{*}$ & $0,042^{*}$ & 0,152 \\
\hline
\end{tabular}

Tabela 5: Comparação de médias dos grupos do estudo.

\begin{tabular}{|l|c|c|c|c|}
\hline & Impulsividade & Autodisciplina & Foco & Escolhas \\
\hline Presencial & 4,478 & 4,418 & 3,374 & 5,275 \\
\hline À distância & 5,551 & 5,087 & 4,000 & 5,377 \\
\hline
\end{tabular}




\section{Teste de correlação de Spearman}

Para a avaliação da relação do autocontrole com os objetivos acadêmicos, utilizou-se o teste de correlação de Spearman. $O$ teste foi escolhido por ser o indicado para situações onde uma das variáveis do estudo é ordinal (HAUKE et al., 2011). Ambos os grupos foram avaliados em conjunto de forma que fosse possível verificar a relação entre as dimensões do autocontrole e o alcance dos objetivos acadêmicos, medido pela média de notas obtidas pelos alunos ao final de um semestre letivo.

Dentre as dimensões do estudo, apenas a dimensão impulsividade não apresentou relação significativa com o aproveitamento acadêmico. Todas as outras dimensões apresentaram relação significativa, o que dá suporte à hipótese 2 . Os achados vão ao encontro dos estudo de Komarraju et al. (2013) e Zhu et al. (2016). Os valores das correlações estão disponíveis na tabela 6.

Tabela 6: Correlação entre autocontrole e aproveitamento acadêmico.

\begin{tabular}{|l|c|c|c|c|}
\hline & Impulsividade & Autodisciplina & Foco & Escolhas \\
\hline Aproveitamento acadêmico & 0,159 & 0,211 & 0,192 & 0,214 \\
\hline Sig. & 0,061 & $\mathbf{0 , 0 1 2}^{*}$ & $\mathbf{0 , 0 2 3}^{*}$ & $\mathbf{0 , 0 1 1}^{*}$ \\
\hline
\end{tabular}

\section{CONCLUSÕES}

A independência, ou autocontrole como está sendo abordado no presente trabalho, é um fator central para o sucesso dos alunos em cursos de ensino à distância (ZHU et al., 2016). Alunos com maiores índices de autocontrole tem maiores chances de alcançar os objetivos acadêmicos e esse fato pode ser percebido no presente estudo. Adicionalmente, alunos de ensino presencial e de ensino à distância mostraram ter diferentes níveis de autocontrole. Em todas as dimensões avaliadas as médias das dimensões de autocontrole dos alunos do ensino à distância foi sempre maior que a dos alunos do ensino presencial, sendo estatisticamente relevantes em três das dimensões, corroborando com o que fora afirmado por Bol et al. (2011).

Para os alunos do ensino presencial, o desafio de caminhar com os estudos é auxiliado pelos mecanismos de controle externos presentes na sala de aula e na faculdade, como horários, chamadas e os próprios colegas de classe, apresentando, assim, uma série de mecanismos de controle formais e informais externos ao aluno (KUHLMANN, 2012). Já no ensino à distância, muitos dos mecanismos de controle não estão presentes, ou se fazem de forma bastante sutil. Assim, é importante enfatizar o papel do autocontrole e utilizar-se de mecanismos que direcionem comportamentos esperados, como incentivos e recompensas, reconhecimento por um bom desempenho e a percepção de que o aluno detém o poder de realizar sua formação.

Adicionalmente, a criação de relações pessoais com a equipe de educadores pode criar um sentimento de pertencimento, aumentando os laços e permitindo que o aluno possa perceber maior apoio no decorrer da sua vida acadêmica para enfrentar os eventuais desafios que se apresentem.

A presente pesquisa foi realizada com alunos de cursos presenciais e à distância, mas levando em consideração o universo de estudantes e a variedade de cursos disponíveis, a pesquisa foi limitada a uma pequena parcela do grupo, sendo necessários maiores esforços de levantamento de participantes de forma 
que as análises estatísticas possam representar a população de alunos presenciais e à distância matriculados nos cursos superiores.

Outro ponto que pode ser percebido como limitação são os cursos participantes. Na presente pesquisa nenhum curso de engenharia se fez presente, assim como cursos de medicina, direito, arquitetura, entre tantos outros, que podem fazer com que ocorram mudanças no perfil do aluno em termos de autocontrole e de alcance dos objetivos acadêmicos.

\section{REFERÊNCIAS}

ARIELY, D.; WERTENBROCH, K.. Procrastination, deadlines, and performance: Self-Control by Precommitment. Psychological science, v.13, n.3, p.219-224, 2002.

BAYNTON, M.. Dimensions of 'Control' in Distance Education: A Factor Analysis. American Journal of Distance Education, v.6, n.2, p.17-31, 1992.

BOL, L.; GARNER, J. K.. Challenges in supporting selfregulation in distance education environments. Journal of Computing in Higher Education, v.23, n.3, p.104-123, 2011.

COSTA, P. T.; MCCRAE, R. R.. Normal Personality Assessment in Clinical Practice: The NEO Personality Inventory.

Psychological Assessment, v.4, n.1, p.5-13 1992.

DIXSON, D. D.. The magic of hope: Hope mediates the relationship between socioeconomic status and academic achievement. Journal of Educational Research, v.111, n.4, p.507-515, 2018.

DUCKWORTH, A. L.. Self-Control and Academic Achievement. Annual Review of Psychology, v.70, p.373-399, 2019.

FERGUSON, C. E.; EL-DIRGHAMI, A.; CHURCHILL, G. A.. Marketing Research: Methodological Foundations. Journal of Marketing Research, v.14, n.1, p.131, 2006.

GIL, A. C.. Métodos das ciências sociais. Métodos e técnicas de pesquisa social, v.264, p.9, 2008.

HAIR, J. F.. Análise Multivariada de Dados. Rio de Janeiro: Bookman, 2009.

HAUKE, J.; KOSSOWSKI, T.. Comparison of Values of Pearson's and Spearman's Correlation Coefficients on the Same Sets of Data. Quaestiones Geographicae, v.30, n.2, p.87-93, 2011.

JACKSON, D. N. Jackson Personality Inventory: Revised (JPIR). Port Huron: 1997.

KINNEAR, T. C.; TAYLOR, J. R.. Marketing research: an applied approach. Nova York: McGraw-Hill, 1987.

KOMARRAJU, M.; NADLER, D.. Self-efficacy and academic achievement: Why do implicit beliefs, goals, and effort regulation matter?. Learning and Individual Differences, v.25, p.67-72, 2013.
KUHLMANN, D.. Governing IT Outsourcing Relationships: The Roles of Contract, Control, and Relational Norms. Hamburg: Diplomica Verlag, 2012.

MALHOTRA, N. K.; BIRKS, D. F.; NUNAN, D. Marketing Research: An Applied Approach. Pearson education, 2017.

NACHAR, N. The Mann-Whitney U: A Test for Assessing Whether Two Independent Samples Come from the Same Distribution. Tutorials in Quantitative Methods for Psychology, v.4, n.1, p.13-20, 2016.

NEVES, L. M.. A contribuição do atendimento de qualidade do POLO EAD redução da evasão de alunos. São Carlos: UFSCar, 2018.

RUXTON, G. D.. The unequal variance t-test is an underused alternative to Student's t-test and the Mann-Whitney U test. Behavioral Ecology, v.17, n.4, 2006.

SCHUNK, D. H.; ZIMMERMAN, B. J.. Motivation and selfregulated learning: Theory, research, and applications. Abingdon: Routledge, 2012.

SILVA, C. G.; FIGUEIREDO, V. F.. Ambiente virtual de aprendizagem: comunicação, interação e afetividade na EAD. Aprenizagem em EAD, v. 1, p.1-16, 2011.

SINGH, K.; MALONEY, T.. Using validated measures of high school academic achievement to predict university success. New Zealand Economic Papers, v.53, n.1, p.89-106, 2019.

SVENSSON, E.. Guidelines to statistical evaluation of data from rating scales and questionnaires. Journal of Rehabilitation Medicine, v.33, n.1, p.47-48, 2001.

TANGNEY, J. P.; BAUMEISTER, R. F.; BOONE, A. L.. High SelfControl Predicts Good Adjustment, Less Pathology, Better Grades, and Interpersonal Success. - Google Scholar. Journal of Personality, v.72, n.2, p.271-324, 2004.

WOLFE, R. N.; JOHNSON, S. D.. Personality as a predictor of college performance. Educational and Psychological Measurement, v.55, n.2, p.177-185, 1995.

ZHU, Y.; AU, W.; YATES, G.. University students' self-control and self-regulated learning in a blended course. Internet and Higher Education, v.30, p.54-62, 2016.

A CBPC - Companhia Brasileira de Produção Científica (CNPJ: 11.221.422/0001-03) detém os direitos materiais desta publicação. Os direitos referem-se à publicação do trabalho em qualquer parte do mundo, incluindo os direitos às renovações, expansões e disseminações da contribuição, bem como outros direitos subsidiários. Todos os trabalhos publicados eletronicamente poderão posteriormente ser publicados em coletâneas impressas sob coordenação da Sustenere Publishing, da Companhia Brasileira de Produção Científica e seus parceiros autorizados. Os (as) autores (as) preservam os direitos autorais, mas não têm permissão para a publicação da contribuição em outro meio, impresso ou digital, em português ou em tradução. 